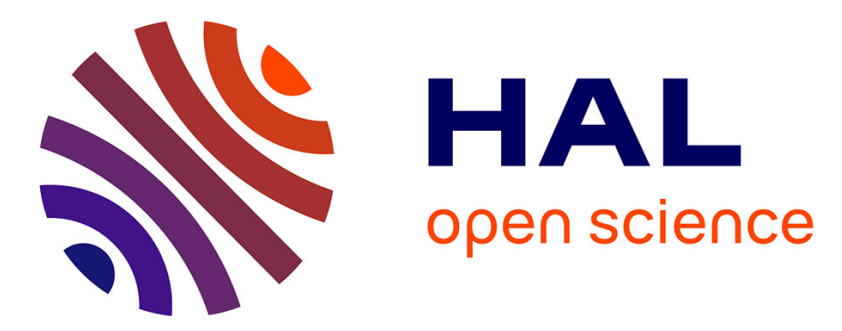

\title{
Method for nonlinear characterization of radio frequency coils made of high temperature superconducting material in view of magnetic resonance imaging applications
}

Olivier Girard, Jean-Christophe Ginefri, Marie Poirier-Quinot, Luc Darrasse

\section{To cite this version:}

Olivier Girard, Jean-Christophe Ginefri, Marie Poirier-Quinot, Luc Darrasse. Method for nonlinear characterization of radio frequency coils made of high temperature superconducting material in view of magnetic resonance imaging applications. Review of Scientific Instruments, 2007, 78 (12), pp.124703. 10.1063/1.2825241. hal-02269965

\section{HAL Id: hal-02269965 https://hal.science/hal-02269965}

Submitted on 23 Aug 2019

HAL is a multi-disciplinary open access archive for the deposit and dissemination of scientific research documents, whether they are published or not. The documents may come from teaching and research institutions in France or abroad, or from public or private research centers.
L'archive ouverte pluridisciplinaire HAL, est destinée au dépôt et à la diffusion de documents scientifiques de niveau recherche, publiés ou non, émanant des établissements d'enseignement et de recherche français ou étrangers, des laboratoires publics ou privés. 


\title{
Method for nonlinear characterization of radio frequency coils made of high temperature superconducting material in view of magnetic resonance imaging applications
}

\author{
Olivier Girard, Jean-Christophe Ginefri, Marie Poirier-Quinot, and Luc Darrasse \\ U2R2M (UMR8081), University Paris-Sud, CNRS, Orsay Cedex 91405, France
}

(Received 1 August 2007; accepted 24 November 2007; published online 28 December 2007)

\begin{abstract}
A contactless method based on reflectometry to accurately characterize an inductive radio frequency (rf) resonator even in the occurrence of a strong electrical nonlinearity is presented. Nonlinear extraction of the unloaded quality factor and resonance frequency is possible by combining an initial low-level swept-frequency calibration with high-level single-frequency measurements. The extraction protocol relies on a simple intrinsic $R, L, C$ model and does not involve a fitting procedure according to a particular nonlinearity model. It includes a correction for strong coupling conditions between the probe and the rf coil, which allows extending the analysis over a wide range of transmitted power. Electrical modeling based on the extracted intrinsic data allows predicting the coil behavior when loaded by any kind of matching network. The method will have implications in different domains such as Magnetic Resonance (MR) applications with superconducting probe heads or analysis of rf properties in nonlinear materials. The method is demonstrated here by characterizing a high temperature superconducting (HTS) coil dedicated to MR imaging at $64 \mathrm{MHz}$. The coil consists in a multiturn spiral design that is self-resonant close to the MR frequency of interest. The $Q$ factor and the resonance frequency are determined as a function of the actual power dissipated in the HTS coil accounting for losses occurring in the measurement system. Further characteristics of the HTS coil are considered in the present paper. The relation between the transmitted power and the magnetic field generated by the coil, which is the most relevant characteristics for MR applications, is directly accessible. The equivalent impedance of the coil under test is also expressed as a function of the total current flowing in the windings. The method could be extended to assess the fundamental properties of the nonlinear material (e.g., the London penetration depth or the critical current density) by including any pertinent model.
\end{abstract}

(C) 2007 American Institute of Physics. [DOI: 10.1063/1.2825241]

\section{INTRODUCTION}

High temperature superconducting (HTS) coils dedicated to magnetic resonance (MR) applications have been shown to significantly enhance the signal to noise ratio, which allows reducing either the size of the elementary detected volume (voxel) or the total scan duration in biomedical MR imaging. ${ }^{1}$ HTS coils have also been applied early in high-resolution MR spectroscopy with typical sensitivity improvements by a factor of $4 .^{2}$ Indeed HTS materials strongly lower the noise contribution coming from the radio frequency (rf) coil since they offer surface resistivities lower than conventional copper by a few orders of magnitude. However, it is well known that HTS devices exhibit nonlinear electrical properties as a function of the applied current density. ${ }^{3}$ This is still an issue since a unified model has not been establish yet to predict the nonlinear behavior over extended temperature and current ranges. ${ }^{4}$ Nonlinearities have been reported in MR imaging applications ${ }^{5,6}$ and can reduce the experimental performance since they are not accounted for in practical implementations. A major consequence is that rf transmission and reception steps can no longer be considered reciprocal as in classical MR (Ref. 6) since the corresponding power levels are different. During transmission of nonrectangular rf pulses, nonlinearity may modify the pulse shape and alter the selective frequency or space excitation profiles. It may also affect the flip angle and the power deposited in the sample, respectively, leading to a misadjusted image contrast and a misevaluated heating hazard. Thus, the determination of the nonlinear characteristics of a given HTS coil is required to preserve the reliability of the diagnosis and the safety of the examination.

The quality factor of the rf coil is a highly relevant parameter in MR applications as it is related to the efficiency for transmission and to the sensitivity of the experiment during reception. ${ }^{7,8}$ Actually it represents the ratio of the stored magnetic energy by the rf coil over the dissipated energy per cycle. The classical method to measure the quality factor on the rf coil tuned at the MR frequency is by using a sweptfrequency analysis of the resonant circuit. As recently pointed out ${ }^{9,10}$ the method is inappropriate to characterize a nonlinear resonant circuit. Indeed the intrinsic electrical parameters vary as the carrier frequency approaches the resonance since the cancellation of the reactance increases the power transmitted to the coil circuit. The change in electrical parameters appears at high power levels as a drop of the quality factor and as a weak change of the resonant fre- 


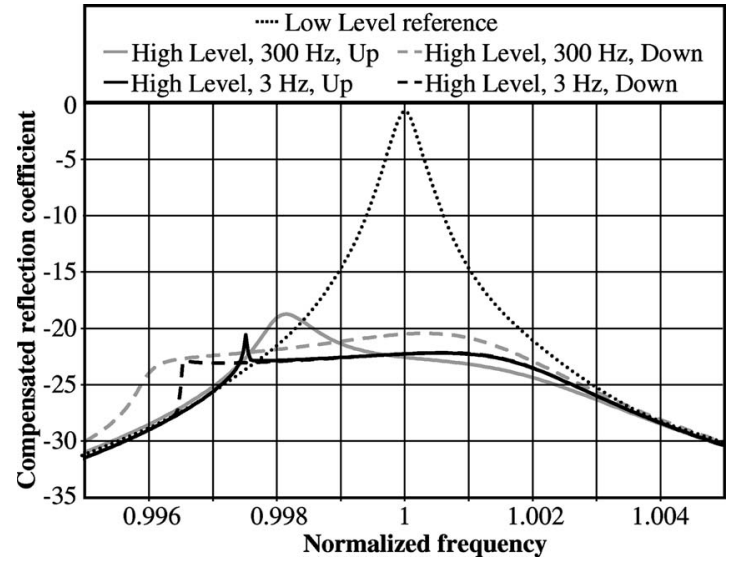

FIG. 1. Swept-frequency analysis of a nonlinear HTS coil performed with the measurement bench of Fig. 2. At low incident power $(-30 \mathrm{dBm})$, a classical Lorentzian shape is observed (doted line) corresponding to a linear behavior of the HTS coil. At high incident power $(+15 \mathrm{dBm})$ huge distortions due to the nonlinear behavior are evidenced: the solid and dashed lines correspond, respectively, to down-up and up-down frequency sweeps; the gray and black lines have been acquired with respective integration filter bandwidths of 300 and $3 \mathrm{~Hz}$ and emphasize complex out-equilibrium effects possibly including long-term thermal drifts.

quency, related to respective increases of the resistivity and of the dynamic inductance of the HTS material. ${ }^{10,11}$ The actual changes cannot be reliably evaluated since the Lorentzian shape classically observed with linear materials is affected by major distortions such as displayed on Fig. 1. It clearly appears that the maximum of the gain response can no more be attributed to the resonance frequency in a nonlinear regime.

Previous attempts to characterize the nonlinear behavior of inductive resonators made of HTS material have been reported, but with inappropriate bandwidth measurement ${ }^{5,12}$ or incomplete assessment of the actual transmitted power. $5,6,12,13$ Such approaches fail to extract the intrinsic nonlinear features. The method described in this paper is an extension of the inductively coupled dual- and single-loop techniques initially proposed for contactless evaluation of linear rf coils. ${ }^{14,15}$ Based on reflectometry at a single frequency, it can be easily implemented and provides a complete rf characterization valid in both linear and nonlinear regimes. The extraction protocol relies on a simple $R, L, C$ model and does not involve a fitting procedure according to a particular nonlinearity model. It includes a correction for strong coupling conditions between the probe and the rf coil, which allows extending the analysis over a wide range of transmitted power.

\section{THEORY}

Consider a measurement bench made of a rf source that powers an inductive probe magnetically coupled to the HTS coil via a bidirectional coupler (Fig. 2) as suggested in the literature. ${ }^{15}$ Here the HTS coil is represented by the equivalent series $R, L, C$ circuit tuned to the pulsation $\omega_{0}$ and having the quality factor $Q$ defined as $L \omega / R$ at the current angular frequency $\omega$. The probe is represented by the equivalent series $R_{P}, L_{P}, C_{P}$ circuit and is intrinsically matched to the impedance $R_{0}$ of the rf source at $\omega_{0}$ (i.e., $R_{P}=R_{0}$ and

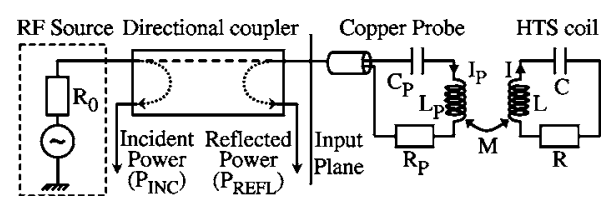

FIG. 2. Modeling of the measurement bench. The nonlinear HTS coil properties are inductively probed by reflectometry using a linear measurement bench.

$\left.L_{P} C_{P} \omega_{0}^{2}=1\right)$. All components are assumed to be ideal ones and the total reactance of the probe circuit to be negligible compared to $R_{0}$ at observed frequencies.

The effect of the magnetic coupling of the rf coil into the probe circuit is equivalent to introducing the additional series impedance $Z_{\text {in }}=M^{2} \omega^{2} / Z$, where $Z$ is the complex impedance of the HTS coil circuit and $M$ the mutual inductance between the two circuits. The reflection coefficient $\rho$ is defined at the input plane from the incident and reflected powers, respectively, $P_{\text {inc }}$ and $P_{\text {refl }}$, as $\left(P_{\text {refl }} / P_{\text {inc }}\right)^{1 / 2}$,

$$
\rho(\omega)=\frac{Z_{P}+Z_{\text {in }}-R_{0}}{Z_{P}+Z_{\text {in }}+R_{0}},
$$

where $Z_{P}$ is the total impedance of the probe circuit.

The dual-reflection measurement ${ }^{15}$ involves a reference data set $\rho_{0}(\omega)$ taken in the absence of the coil $(M=0)$ and defines the compensated reflection coefficient as $\rho_{\text {comp }}(\omega)$ $=\rho(\omega)-\rho_{0}(\omega)$.

In the linear regime, $R$ has a constant value and $\rho_{\text {comp }}(\omega)$ is independent of the incident power level. A classical sweptfrequency analysis of $\left|\rho_{\text {comp }}(\omega)\right|$ allows to extract $Q$,

$$
Q \underset{\substack{\text { linear } \\ \text { regime }}}{=} \frac{\omega_{0} / \mathrm{BW}_{-3 \mathrm{~dB}}}{1-\rho_{\text {comp }}\left(\omega_{0}\right)}
$$

where $\mathrm{BW}_{-3 \mathrm{~dB}}$ is the $3 \mathrm{~dB}$ attenuation bandwidth measured on the $\left|\rho_{\text {comp }}(\omega)\right|$ frequency response. As compared to the quality factor usually estimated by $\omega_{0} / \mathrm{BW}_{-3 \mathrm{~dB}}$, the denominator in Eq. (2) represents a correction factor accounting for the power dissipated in the probing system. Without correction the extra dissipated power would lead to an underestimated apparent quality factor. Using any arbitrarily strong coupling condition, the correction is equivalent to measuring $Q$ under the weak coupling condition $\left[\rho_{\text {comp }}\left(\omega_{0}\right) \approx 0\right]$.

When the HTS coil is driven at high incident power levels and enters the nonlinear regime, $R$ is no more constant along a frequency sweep since the power transmitted to the coil varies across the resonance. In addition, the HTS coil inductance may slightly vary due to a change of the London penetration depth. ${ }^{11}$ A new derivation of $Q$, no more based on the previous swept-frequency analysis and accounting for simultaneous changes of resistance and reactance, is then needed.

The following expression: $Z_{\text {in }}=M^{2} \omega^{2} / Z$ $=2 R_{0} \rho_{\text {comp }}(\omega) /\left[1-\rho_{\text {comp }}(\omega)\right]$, gives the real and imaginary parts of the HTS coil impedance $Z$ as a function of the modulus and the phase $\left[\angle \rho_{\text {comp }}(\omega)\right]$ of $\rho_{\text {comp }}(\omega)$, respectively,

$$
R(\omega)=M^{2} \omega^{2} \frac{\cos \left[\angle \rho_{\mathrm{comp}}(\omega)\right]-\left|\rho_{\mathrm{comp}}(\omega)\right|}{2 R_{0}\left|\rho_{\mathrm{comp}}(\omega)\right|},
$$




$$
X(\omega)=-M^{2} \omega^{2} \frac{\sin \left[\angle \rho_{\text {comp }}(\omega)\right]}{2 R_{0}\left|\rho_{\text {comp }}(\omega)\right|} .
$$

Both $L$ and $M$ may be assumed to be almost constant since the redistribution of the supercurrent occurs at a small scale compared to the HTS coil and probe dimensions. A variation of the HTS coil reactance resulting from a slight change of $L$ can be probed using Eq. (4). Moreover, the change $\Delta X$ in reactance can be used to monitor the resonance frequency shift due to the change of inductance $\Delta L$,

$$
\Delta \omega_{0} \approx-\frac{1}{2} \frac{\Delta X}{L}=-\frac{1}{2} \frac{\Delta L}{L} \omega_{0} .
$$

The three above equations indicate that single-frequency measurements of $Q$ and $\Delta \omega_{0}$ are possible once $L / M^{2}$ is known. The latter value is easily estimated from a single low-level swept-frequency measurement since both above derivations of $Q$ are applicable in the linear regime. As long as the probe is maintained at a fixed position relatively to the HTS coil, the low-level calibration can be used as the preliminary step of a nonlinear characterization of the HTS coil using single-frequency measurements at higher levels.

The power dissipated in the HTS coil $P_{\text {coil }}=R|I|^{2}$ is evaluated as

$$
\begin{aligned}
P_{\text {coil }}(\omega)= & \operatorname{Re}\left(Z_{\text {in }}\right)\left|I_{P}\right|^{2}=2 P_{\text {inc }}\left|\rho_{\text {comp }}(\omega)\right|\left\{\cos \left[\angle \rho_{\text {comp }}(\omega)\right]\right. \\
& \left.-\left|\rho_{\text {comp }}(\omega)\right|\right\} .
\end{aligned}
$$

By combining Eqs. (3)-(6), the nonlinearity of the HTS coil can then be expressed either directly in the form of a $\left(Q, P_{\text {coil }}\right)$ and $\left(\omega_{0}, P_{\text {coil }}\right)$ characteristics, or alternatively as a $(R,|I|)$ and $(X,|I|)$ characteristics when the value of $L$ is known.

To implement MR experiments, the dependence of the rf magnetic field $B_{1}$ generated by the HTS coil as a function of the applied power is required. In opposition to the characterization steps above, the amplitude and phase of $B_{1}$ can be derived from $\rho_{\text {comp }}$ measurements without a low-level calibration, according to

$$
B_{1}^{S_{P}}(\omega)=\frac{M I_{C}}{S_{P}}=\frac{2 \rho_{\mathrm{comp}}(\omega)}{\omega S_{P}} \sqrt{2 R_{0} P_{\mathrm{inc}}},
$$

where $B_{1}^{S_{P}}$ is the average $\mathrm{rf}$ magnetic field through $S_{P}$, the equivalent area of the probe. Assuming a negligible deformation of the $B_{1}$ field, it is possible to derive the local nonlinear characteristics $\left(B_{1}, P_{\text {coil }}\right)$ from the spatially averaged $\left(B_{1}^{S_{P}}, P_{\text {coil }}\right)$ characteristics,

$$
B_{1}=\frac{B_{1 \text { ref }}}{B_{1}^{S_{P}} B_{1}^{S_{P}}}
$$

where $B_{1 \text { ref }}$ and $B_{1}^{S_{P}}$ ref are single data points measured at the same power $P_{\text {coil ref }}$ level. $B_{1 \text { ref }}$ and $P_{\text {coil ref }}$ may be extracted in situ by MR using a standard rf-pulse calibration. ${ }^{7}$ In the actual MR experimental setup with a particular coiltransmitter matching network, the power transmitted to the HTS coil has to be monitored in order to account for the variable power reflection. Alternatively, on-the-bench local measurement of $B_{1}$ is achievable by using a small probe
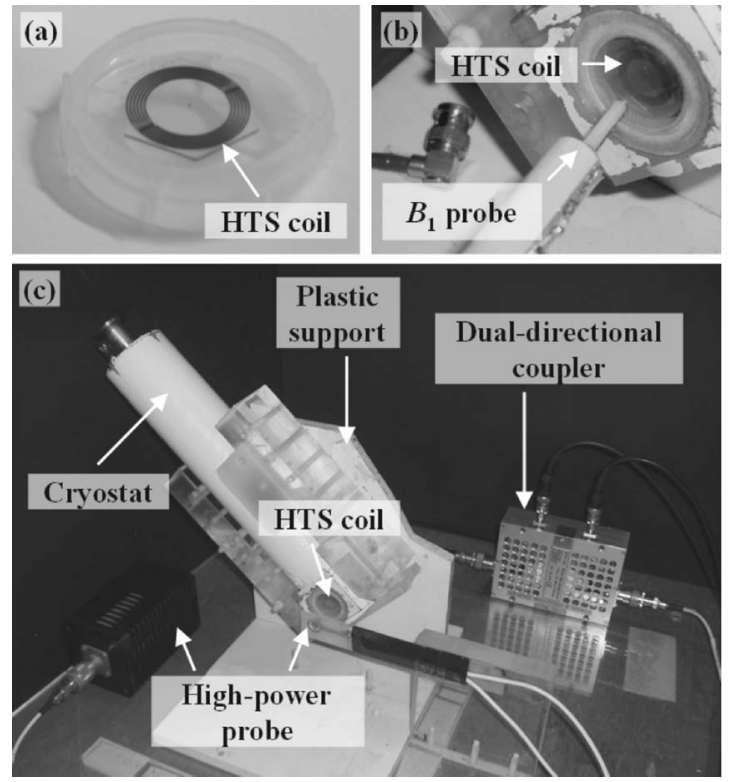

FIG. 3. Experimental setup. The HTS coil (a) is a multiturn transmission line resonator (Ref. 16). The pickup coil is used to probe local $B_{1}$ (b). The high-power setup (c) involves a dedicated probe made of a single-turn copper-wire loop and a high-power $50 \Omega$ load connected using a coaxial cable. The probe is connected to the rf amplifier and the multireceiver MR console through the dual-directional coupler.

making negligible the variation of $B_{1}$ across $S_{P}$. Such a small probe allows fully characterizing the HTS coil by bench measurement. However, a larger probe may be preferred to investigate high levels of $P_{\text {coil }}$, since it can provide a stronger coupling coefficient and thus a larger power transfer to the HTS coil.

\section{EXPERIMENT}

The experimental setup and the HTS coil are displayed on Fig. 3. The HTS coil characterized here is a $12 \mathrm{~mm}$ diameter multiturn transmission line resonator ${ }^{16}$ made of $\mathrm{YBaCuO}$ deposited on a sapphire $\mathrm{r}$-cut dielectric substrate (THEVA, Ismaning, Germany) and incorporated in a cryogenic setup suitable for proton micro-MRI at $1.5 \mathrm{~T}$ (64 MHz). The HTS coil is maintained at an actual temperature of about $80 \mathrm{~K}$. A single-turn rf coil of same diameter as the HTS coil, but made of copper instead of $\mathrm{YBaCuO}$ and tuned by a $250 \mathrm{pF}$ chip capacitor, was investigated in order to check the validity of the single-frequency characterization.

The probe used for the nonlinear extraction at highpower level is a single-turn copper-wire loop $(\varnothing 12 \mathrm{~mm}$, same diameter as the HTS coil) tuned to $64 \mathrm{MHz}$ with a $150 \mathrm{pF}$ capacitor and matched to the rf source impedance using an $R_{P}$ value of $50 \Omega$. The actual $R_{P}$ is implemented using a $\lambda / 2$ coaxial cable terminated by a high-power $50 \Omega$ load. The attenuation and phase delay introduced by the transmission line of the probe have been measured and are corrected in the $\rho$ measurements.

The low-power swept-frequency analysis is performed using a network analyzer (Agilent E5061A, USA) based on its own integrated dual-directional coupler.

The high-power measurements are done in a pulsed mode with a multireceiver MR console (Tecmag Apollo, 
Houston, TX, USA), a $2 \mathrm{~kW}$ rf amplifier (ENI MRI 2000, Rochester, NY, USA) and a $-40 \mathrm{~dB}$ dual-directional coupler (RFPA RFC 001400-40-600, Artigues Pres Bordeaux, France). The complete setup including the MR console and the directional coupler is calibrated at the input plane following a classical short/open/load procedure according to a oneport error model. ${ }^{17}$ The $\mathrm{rf}$ pulses have a rectangular shape with $2 \mathrm{~ms}$ duration and are periodically applied with a repetition time $T_{R}$. The value of $T_{R}$ must be kept sufficiently long to avoid thermal drifts. The actual duty cycle corresponding to nonobservable drifts does not exceed $1 \%$ at the highest power levels. The incident and reflected waves are digitally sampled at a dwell time of $3 \mu$ s and averaged over a series of hundred rf pulses during the postprocessing step. A hundred samples at the center of each averaged pulse are considered in order to give the mean and standard deviation of the measured $\rho$. Error bars on the results are estimated with the error propagation model developed in Appendix, taking the mean and standard deviation of measured $P_{\text {inc }}$, $\left|\rho_{\text {comp }}\right|$, and $\angle \rho_{\text {comp }}$, plus a $0.05 \mathrm{~dB}$ uncertainty affecting the correction for attenuation in the transmission line of the probe.

For the bench measurement of local $B_{1}$, the pickup coil consists in a single-turn copper-wire loop wrapped around a $3 \mathrm{~mm}$ diameter nylon mandrel, tuned to the MR frequency with an $80 \mathrm{pF}$ capacitor and matched to the receiver by inserting a $47 \Omega$ series resistance. $B_{1 \text { ref }}$ is measured along the coil axis at $2 \mathrm{~mm}$ from the coil plane which is the minimum distance needed for the cryostat insulation. Some inaccuracy may be due to the inexact knowledge of the loop area and of the actual position of the HTS coil in the cryogenic setup. Such systematic errors are not reported in the results since they do not affect the shape of the nonlinearities and could be eliminated by using further calibration procedures.

The direct measurement of $L$ at the HTS coil ends is not accessible with the present experimental setup. Instead, the bench measurement of $B_{1}$ ref is combined with a Biot-Savard computation of the $B_{1} / I$ ratio, assuming the multiturn HTS coil geometry with an infinitesimally thin wire and negligible propagation effects. Estimated values of $I, R$, and $L$ are then deduced from simultaneous measurements of $B_{1}$ ref, $P_{\text {coil }}$, and $Q$.

\section{RESULTS}

\section{Confidence test with the copper rf coil}

The copper rf coil exhibits a $Q$ of 91 by swept-frequency analysis using a low coupling condition $\left[\rho_{\text {comp }}\left(\omega_{0}\right)\right.$ of $-31 \mathrm{~dB}]$, a narrow integration filter $(100 \mathrm{~Hz}$ bandwidth), and a RF source level of $+10 \mathrm{dBm}$. From this reference value of $Q$, a confidence interval of $[-2.5 \%,+1 \%]$ is observed with the pulsed single-frequency measurement over a domain of coupling conditions $\left[\rho_{\text {comp }}\left(\omega_{0}\right)\right.$ of -30 to $\left.-5 \mathrm{~dB}\right]$ and of incident power levels $(-25$ to $+50 \mathrm{dBm})$.

The extracted value of $L$ is $26 \mathrm{nH}$ from the local $B_{1}$ measured over a range of $2-15 \mathrm{~mm}$ distances along the copper rf-coil axis. This is in agreement within less than $3 \%$ error compared to the value obtained from a combined
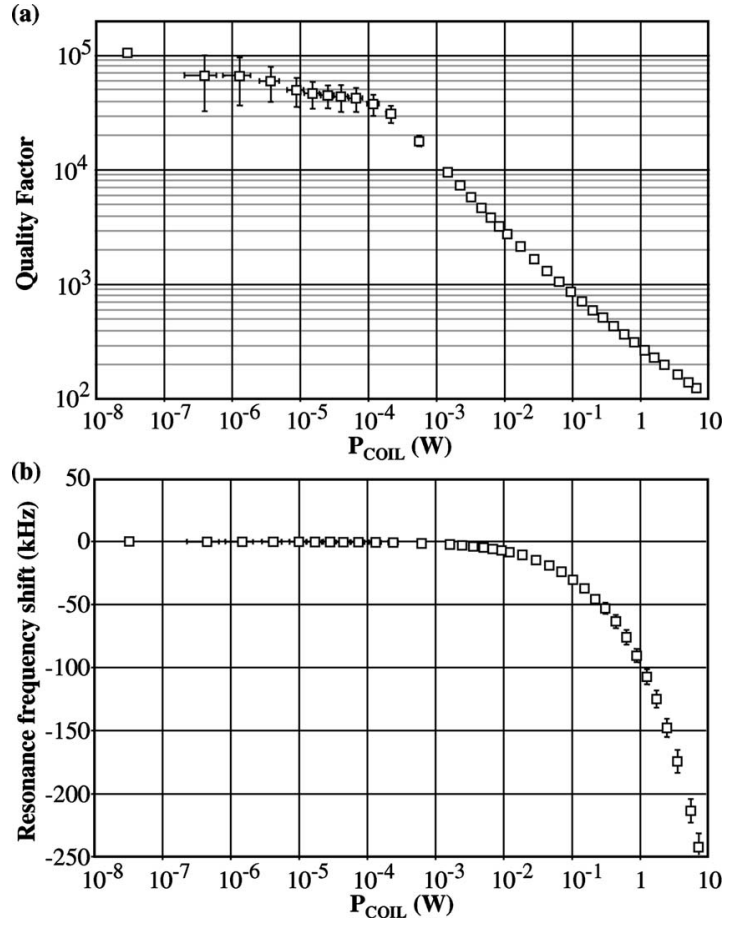

FIG. 4. Experimental quality-factor drop (a) and resonance-frequency shift (b) expressed as a function of the power dissipated in the HTS coil. The low-level resonance frequency setting the shift reference is $63910 \mathrm{MHz}$. The lowest-power data point is obtained in the linear regime using sweptfrequency analysis under weak coupling condition, whereas higher-power data points are extracted from single-frequency measurements under strong coupling conditions.

measurement of the resonance frequency and the tuning capacitor.

\section{Nonlinear characterization of the HTS coil}

The pulsed characterization using a frequency of 63.910 MHz and an incident power range of $-20-50 \mathrm{dBm}$ leads to the $\left(Q, P_{\text {coil }}\right)$ and $\left(\Delta \omega_{0}, P_{\text {coil }}\right)$ characteristics of the HTS coil displayed on Fig. 4. This frequency corresponds within $\pm 0.1 \mathrm{kHz}$ to the low-level resonance observed by swept-frequency calibration under strong coupling conditions $\quad\left[P_{\text {inc }}=-45 \mathrm{dBm}, \quad \rho_{\text {comp }}\left(\omega_{0}\right)=-0.13 \pm 0.01 \mathrm{~dB} \quad\right.$ and $\left.\omega_{0} / \mathrm{BW}_{-3 \mathrm{~dB}}=1270 \pm 10\right]$. The lowest-power $Q$ data point of $107000 \pm 3 \%$ is observed using the swept-frequency analysis performed in the low coupling condition $\left[\rho_{\text {comp }}\left(\omega_{0}\right)\right.$ of $-36 \mathrm{~dB}]$, with a narrow integration filter $(10 \mathrm{~Hz}$ bandwidth) and a RF source level of $-30 \mathrm{dBm}$. Up to this power level, not actually shown in the figure, neither the quality factor nor the resonance frequency exhibits significant variations indicating that the HTS coil behaves linearly. A strongly nonlinear behavior is evidenced on the $\left(Q, P_{\text {coil }}\right)$ characteristics above $10^{-4} \mathrm{~W}$. Up to this level, the resonance frequency stays at $63.910 \mathrm{MHz} \pm 1 \mathrm{kHz}$. Above $10^{-4} \mathrm{~W}$ it decreases progressively until reaching about $-230 \mathrm{kHz}$ at the highest power of $7 \mathrm{~W}$.

Figure 5 displays the $(R,|I|)$ and $(X,|I|)$ characteristics based on the previous data set combined with a measured $L$ of $4.5 \pm 0.8 \mu \mathrm{H}$. The values of $R$ and $X$ at the lowest-power level are $17 \pm 0.5 \mathrm{~m} \Omega$ and $0 \pm 10^{-4} \Omega$, respectively. The strong nonlinear regime is observed above $0.05 \mathrm{~A}$ with a 


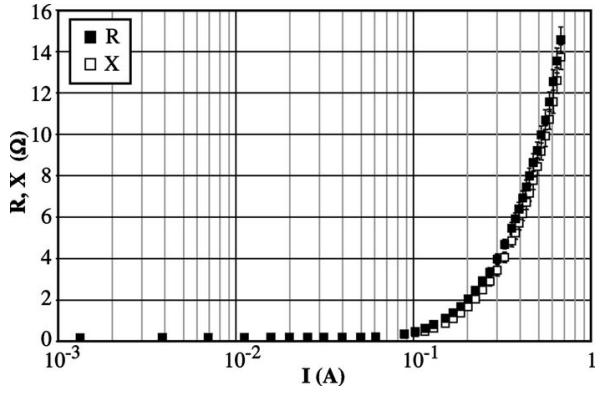

FIG. 5. Experimental real $(R)$ and imaginary $(X)$ parts of the HTS coil equivalent impedance expressed as a function of the current flowing in the windings. These characteristics are extracted with $L=4.5 \mu \mathrm{H}$ and the same data set as in Fig. 3.

power-law exponent progressively decreasing from 2.9 to about 1.5 at the highest current value of $0.7 \mathrm{~A}$. Within an intermediate weakly nonlinear regime up to $0.05 \mathrm{~A}$, also apparent on $\left(Q, P_{\text {coil }}\right)$ characteristic, both $((R,|I|)$ and $(X,|I|)$ characteristics follow an average increase with a 0.3 power law.

Figure 6 displays the magnitude and phase of $B_{1}$ as a function of $P_{\text {coil }}$. The response of an ideal linear circuit, that would keep the low-level electrical properties of the HTS coil at any dissipated power level, is also plotted as a reference. As expected, the saturation of the HTS material close to its critical current tends to limit the rf magnetic field generated at high power. A $B_{1}$-phase deviation also appears at high power in relation to the resonance frequency shift of the HTS coil.

\section{DISCUSSION}

A contactless method based on reflectometry has been demonstrated to accurately characterize an inductive rf resonator even in the occurrence of a strong nonlinearity. Nonlinear extraction of the unloaded quality factor and resonance frequency is possible by combining an initial low-level swept-frequency calibration with high-level single-frequency measurements. Since the method allows investigating the

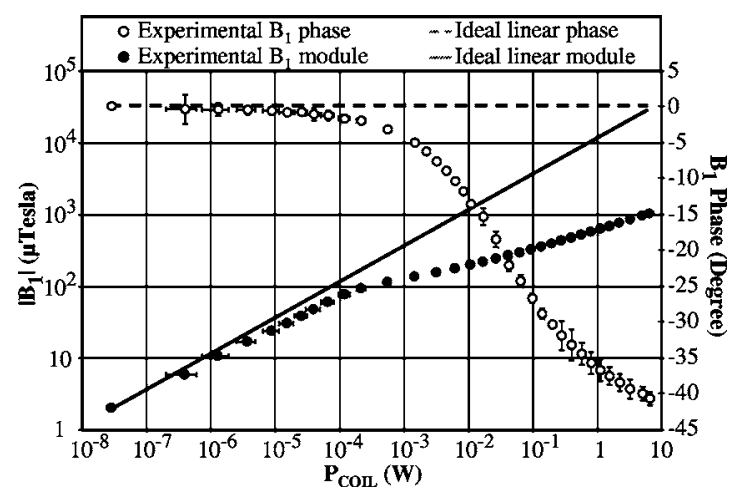

FIG. 6. Experimental amplitude (filled data points) and phase (empty data points) as a function of the power dissipated in the HTS coil. The amplitude is for $2 \mathrm{~mm}$ from the center along the coil axis. This characteristic is extracted from the same data set as in Fig. 3. For comparison solid (amplitude) and dashed (phase) lines represent the ideal linear case. nonlinear regime with a pulsed power application, varying the rf-pulse duration and duty cycle can also help assessing thermal effects.

Compared to the previous investigations with nonlinear inductive $\mathrm{rf}$ resonators, $5,6,12,13$ the present characterization is based on complete understanding of the incident and reflected rf power as a function of frequency. The nonlinear behavior is expressed as a function of the actual power dissipated in the resonator, accounting for the absorption in the measurement probe at any coupling strength. A very large range of transmitted power is accessible by varying the coupling conditions. For accuracy purpose, the measurement probe impedance has been matched to the rf source, making the whole system nearly matched either in strong coupling conditions when the coil is highly resistive, i.e., at highpower level, or in weak coupling conditions. As compared to an unmatched probe, this results in a larger modification of the reflected power due the presence of the rf resonator over a broad range of coupling conditions.

The method will have implications in different domains such as MR applications with superconducting probe heads or analysis of $\mathrm{rf}$ properties in nonlinear materials.

For MR applications, the method provides a direct extraction of the intrinsic ratio $B_{1} / \sqrt{P_{\text {coil }}}$, which quantifies both the rf-coil efficiency for transmission and its sensitivity for reception. ${ }^{7}$ Once the intrinsic behavior of the HTS coil is known, it can be taken into account to design the most appropriate matching circuit for practical implementation minimizing nonlinear effects. Furthermore the accurate prediction of $B_{1}$ can be involved to correct the local flip angle errors during excitation, including phase and pulse-shape distortions. Finally, elaboration of more robust rf coils based on improved materials or different coil geometries can be considered.

Orders of magnitude for practical MR imaging experiments are a maximum transmit $B_{1}$ amplitude of about $60 \mu \mathrm{T}$, which corresponds to a shortest $90^{\circ}$ pulse duration of $100 \mu \mathrm{s}$, and a received signal amplitude given by $E=\omega_{0} \mu_{0} M_{0} S / 2=R I$. The electromotive force $E$ is estimated in the case of a receiver loop of effective area $S$ applied onto the surface of a half-infinite sample of uniform magnetization $M_{0}$. Taking $M_{0}$ of $0.0047 \mathrm{~A} / \mathrm{m}$ for a water sample at $1.5 \mathrm{~T}$ and $S$ of $0.0014 \mathrm{~m}^{2}, E$ is about $1.6 \mathrm{mV}$. This corresponds to $I=38 \mathrm{~mA}$ and $P_{\text {coil }}=6.10^{-5} \mathrm{~W}$ according to the $(R,|I|)$ characteristics of Fig. 5. Such data show that the HTS coil stays in the weakly nonlinear regime described above, keeping a huge quality factor for MR reception. In this regime the resonance frequency shift stays within $1 \mathrm{kHz}$, which corresponds to less than $2^{\circ}$ of phase deviation, i.e., a nonsignificant MR signal distortion. For the $B_{1}$-amplitude range investigated in these measurements, much larger frequency shifts of up to $230 \mathrm{kHz}$ have been observed. However, according to the correspondingly lower quality factors, the maximum phase deviation does not exceed $45^{\circ}$ and stays still lesser than $2^{\circ}$ at the nominal level of $60 \mu \mathrm{T}$. Some specific MR sequences critically sensitive to the transmit or receive phase might require a phase correction based on the present method. Moreover, the nuclear spins can induce in the HTS coil a RF power level comparable to the rf- 
transmission one. This evidences a potential radiationdamping issue that should be solved, e.g., using dedicated electronics. ${ }^{18}$ In practice, phase deviation and radiation damping raised no problematic issues with the basic MRmicroimaging implemention at $1.5 \mathrm{~T}$ (Ref. 16) based on an overcoupled double-tuned matching network scheme. ${ }^{19}$

In the material science domain, complex models have been developed to assess fundamental properties of HTS materials, e.g., critical current, penetration depth, and kinetics inductance, by characterizing the surface impedance of resonant HTS devices. ${ }^{10,11}$ The impedance $Z$ of the resonator measured by the present method could be directly related to the actual surface impedance by applying a geometric factor accounting for the surface current distribution. The present data suggest that both weakly and strongly nonlinear regimes are explored, which remain to be closely interpreted according to pertinent nonlinearity models, outside the scope of this paper. Roughly, the current in the HTS coil appears to be asymptotically limited at about $1 \mathrm{~A}$. Assuming a uniform distribution over the $200 \mu \mathrm{m}$ width and $300 \mathrm{~nm}$ thickness of the HTS film, it would correspond to a current-density magnitude of the order of $2 \times 10^{6} \mathrm{~A} / \mathrm{cm}^{2}$, in fair agreement with manufacturer's specification.

Analysis of intrinsic film properties from the present inductive characterization of a RF resonator can be improved since it relies on both simple assumptions of a $B_{1} / I$ distribution derived from a Biot-Savard computation and of a constant value of the equivalent capacitance $C$. The former assumption might introduce a scaling error on $R, X$, and $I$. The latter one ignores local saturation effects where the redistribution of the supercurrent would change $C$ and $L$ simultaneously. Involving a full electromagnetic computation would help to get a more accurate characterization model. Another way to discriminate for inductive and capacitive shifts could be to apply the present method using several different carrier frequencies.

\section{ACKNOWLEDGMENTS}

The authors want to thank Dr. Paul Crozat for helpful discussions. This work was granted by Guerbet Research (Roissy-Charles de Gaulle, France), by the French Ministry of Research in the frame of the RNTS Program and by the IPA Joint Program from CNRS and CEA.

\section{APPENDIX: ERROR PROPAGATION MODEL}

For clarity in this section $\rho_{\text {comp }}\left(\omega_{0}\right)$, with $\omega_{0}$ the lowlevel resonance pulsation is simply noted $\rho_{\text {comp. }}$.

\section{Complete expression of the quasiconstant term $L / M^{2}$}

$L / M^{2}$ is estimated from the linear regime on-resonance $\rho_{\text {comp }}^{\text {linear }}$ and $Q_{\text {app }}=\omega_{0} / \mathrm{BW}_{-3 \mathrm{~dB}}$ measurements as

$$
\frac{L}{M^{2}}=\omega_{0} \frac{Q_{\text {app }}}{2 R_{0} \rho_{\text {comp }}}
$$

\section{$Q$ factor and frequency-shift extraction}

The quality factor of the coil is extracted from Eq. (3), $Q=L \omega / R$, and the value of $L / M^{2}$. The complete extraction formula is given by

$$
Q=\frac{Q_{\text {app }}}{\rho_{\text {comp }}^{\text {linear }}} \frac{\left|\rho_{\text {comp }}\right|}{\cos \left[\angle \rho_{\text {comp }}\right]-\left|\rho_{\text {comp }}\right|} .
$$

The logarithmic derivative is used to deduce the total relative error on $Q$,

$$
\begin{aligned}
\frac{\delta Q}{Q}= & \frac{\delta Q_{\text {app }}}{Q_{\text {app }}}+\frac{\delta \rho_{\text {comp }}^{\text {linear }}}{\rho_{\text {comp }}}+\frac{\delta\left|\rho_{\text {comp }}\right|}{\left|\rho_{\text {comp }}\right|} \frac{\cos \left[\angle \rho_{\text {comp }}\right]}{\cos \left[\angle \rho_{\text {comp }}\right]-\left|\rho_{\text {comp }}\right|} \\
& +\frac{\delta \angle \rho_{\text {comp }}}{\angle \rho_{\text {comp }}}\left|\frac{\angle \rho_{\text {comp }} \sin \left[\angle \rho_{\text {comp }}\right]}{\cos \left[\angle \rho_{\text {comp }}\right]-\left|\rho_{\text {comp }}\right|}\right| .
\end{aligned}
$$

A similar approach leads to

$$
\begin{aligned}
\frac{\delta \Delta \omega_{0}}{\Delta \omega_{0}}= & \frac{\delta(\Delta X / L)}{\Delta X / L}=\frac{\delta \omega_{0}}{\omega_{0}}+\frac{\delta Q_{\text {app }}}{Q_{\text {app }}}+\frac{\delta \rho_{\text {comp }}^{\text {linear }}}{\rho_{\text {comp }}^{\text {linear }}}+\frac{\delta\left|\rho_{\text {comp }}\right|}{\left|\rho_{\text {comp }}\right|} \\
& +\frac{\delta \angle \rho_{\text {comp }}}{\angle \rho_{\text {comp }}} \frac{\angle \rho_{\text {comp }}}{\tan \left[\angle \rho_{\text {comp }}\right]} .
\end{aligned}
$$

\section{Power extraction}

The logarithmic derivative of Eq. (6) directly gives the error on $P_{\text {coil }}$,

$$
\begin{aligned}
\frac{\delta P_{\text {coil }}}{P_{\text {coil }}}= & \frac{\delta P_{\text {inc }}}{P_{\text {inc }}}+\frac{\delta\left|\rho_{\text {comp }}\right|}{\left|\rho_{\text {comp }}\right|}\left|\frac{\cos \left[\angle \rho_{\text {comp }}\right]-2\left|\rho_{\text {comp }}\right|}{\cos \left[\angle \rho_{\text {comp }}\right]-\left|\rho_{\text {comp }}\right|}\right| \\
& +\frac{\delta \angle \rho_{\text {comp }}}{\angle \rho_{\text {comp }}}\left|\frac{\angle \rho_{\text {comp }} \sin \left[\angle \rho_{\text {comp }}\right]}{\cos \left[\angle \rho_{\text {comp }}\right]-\left|\rho_{\text {comp }}\right|}\right| .
\end{aligned}
$$

\section{Magnetic field extraction}

Starting from Eq. (7) the following expression is derived:

$$
\frac{\delta\left|B_{1}\right|}{\left|B_{1}\right|}=\frac{1}{2}\left[\frac{\delta P_{\text {inc }}}{P_{\text {inc }}}+\frac{\delta R_{0}}{R_{0}}\right]+\frac{\delta S_{P}}{S_{P}}+\frac{\delta\left|\rho_{\text {comp }}\right|}{\left|\rho_{\text {comp }}\right|}+\frac{\delta \omega_{0}}{\omega_{0}}
$$

and

$$
\frac{\delta \angle B_{1}}{\angle B_{1}}=\frac{\delta \angle \rho_{\text {comp }}}{\angle \rho_{\text {comp }}} .
$$

\section{Further extraction using the inductance of the HTS coil}

The relative errors affecting $R$ and $|I|$ are given below,

$$
\begin{aligned}
\frac{\delta R}{R}= & \frac{\delta Q}{Q}+\frac{\delta L}{L}+\frac{\delta \omega_{0}}{\omega_{0}}, \\
\frac{\delta|I|}{|I|}= & \frac{1}{2}\left[\frac{\delta P_{\text {inc }}}{P_{\text {inc }}}+\frac{\delta Q_{\text {app }}}{Q_{\text {app }}}+\frac{\delta \rho_{\text {comp }}^{\text {linear }}}{\rho_{\text {comp }}^{\text {linear }}}+\frac{\delta L}{L}+\frac{\delta \omega_{0}}{\omega_{0}}\right] \\
& +\frac{\delta\left|\rho_{\text {comp }}\right|}{\left|\rho_{\text {comp }}\right|} .
\end{aligned}
$$

Looking at the expressions concerning $Q, P_{\text {coil }}$, and $R$ it is clear that the measurement relative error $\delta\left|\rho_{\text {comp }}\right| /\left|\rho_{\text {comp }}\right|$ 
will be amplified for $\left|\rho_{\text {comp }}\right| \approx 1$ when $\angle \rho_{\text {comp }} \approx 0$ due to the $\cos \left[\angle \rho_{\text {comp }}\right]-\left|\rho_{\text {comp }}\right|$ term, which is the case for strong coupling at low power level on resonance. This explains the relatively high error bars represented on Figs. 3 and 5. On the other side the method is particularly accurate for high power level extraction. Indeed it corresponds to $\left|\rho_{\text {comp }}\right| \approx 0$ for which no relative error amplification occurs.

${ }^{1}$ L. Darrasse and J.-C. Ginefri, Biochimie 85, 915 (2003).

${ }^{2}$ H. D. W. Hill, IEEE Trans. Appl. Supercond. 7, 3750 (1997).

${ }^{3}$ Z. Shen, High-temperature Superconducting Microwave Circuits (Artech House, Boston, 1994)

${ }^{4}$ S. M. Anlage, W. S. Hu, C. P. Vlahacos, D. Steinhauer, B. J. Feenstra, S. K. Dutta, A. Thanawalla, and F. C. Wellstood, J. Supercond. 12, 353 (1999).

${ }^{5}$ J. Miller, S. Hurlston, Q. Ma, D. Face, D. Kountz, J. Macfall, L. Hedlund, and G. Johnson, Magn. Reson. Med. 41, 72 (1999).

${ }^{6}$ R. D. Black, T. A. Early, and G. A. Johnson, J. Magn. Reson., Ser. A 113,
74 (1995).

${ }^{7}$ D. Hoult and R. Richards, J. Magn. Reson. 24, pp. 71-85 (1976).

${ }^{8}$ W. Edelstein, G. Glover, C. Hardy, and R. Redington, Magn. Reson. Med. 3, 604 (1986).

${ }^{9}$ P. J. Petersan and S. M. Anlage, J. Appl. Phys. 84, 3392 (1998).

${ }^{10}$ J. C. Booth, K. Leong, S. A. Schima, C. Collado, J. M. O'callaghan, and J. Mateu, J. Supercond. Novel Magn. 19, 531 (2006).

${ }^{11}$ D. E. Oates, J. Supercond. Novel Magn. 20, 3 (2007).

${ }^{12}$ K. Chan, K. Lee, B. Yan, E. Gao, G. Shen, Q. Ma, and E. Yang, Proceedings of the Tenth Annual Meeting of the ISMRM, 2002 (unpublished).

${ }^{13}$ S. Hurlston, S. Suddarth, A. Nugent, and G. Johnson, Proceedings of the Seventh Annual Meeting of the ISMRM, 1999 (unpublished).

${ }^{14}$ L. Darrasse and G. Kassab, Rev. Sci. Instrum. 64, 1841 (1993).

${ }^{15}$ J. Ginefri, E. Durand, and L. Darrasse, Rev. Sci. Instrum. 70, 4730 (1999).

${ }^{16}$ J. Ginefri, L. Darrasse, and P. Crozat, Magn. Reson. Med. 45, 376 (2001).

${ }^{17}$ W. S. Su and S. M. Riad, IEEE Trans. Instrum. Meas. 42, 157 (1993).

${ }^{18}$ L. Picard, M. Vonkienlin, and M. Decorps, J. Magn. Reson., Ser. A 117, 262 (1995).

${ }^{19}$ A. Raad and L. Darrasse, Magn. Reson. Imaging 10, 55 (1992). 